\title{
Multiple miliare Osteome des Gesichtes
}

\author{
Multiple Miliary Osteoma of the Face
}

Autoren

Institut

\section{N. Adler, A. Wagner, C. Bayerl}

Klinik für Dermatologie und Allergologie, Städtische Kliniken Wiesbaden HSK, Wilhelm-Fresenius-Klinik GmbH

\section{Bibliografie}

DOI 10.1055/s-2007-966357

Akt Dermatol 2007; 33:

164-165 @ Georg Thieme

Verlag KG Stuttgart · New York ISSN 0340-2541

Korrespondenzadresse

\section{Nicole Adler}

Klinik für Dermatologie und Allergologie

Städtische Kliniken Wiesbaden Lehrkrankenhaus der

Universität Mainz

HSK, Wilhelm-Fresenius-Klinik

$\mathrm{GmbH}$

Aukammallee 39

65191 Wiesbaden

nic.adler@gmx.de

\section{Zusammenfassung \\ $\nabla$}

Eine 45-jährige Frau stellte sich mit multiplen kleinen knöchernen Strukturen in der Haut im Stirnbereich, welche seit Jahren bestünden, vor. In ihrer Jugend litt sie unter einer Akne vulgaris. Histologisch konnte die Diagnose der miliaren Osteome gesichert werden.

Osteoma cutis ist charakterisiert durch eine Formation von kompaktem Knochen in der Haut.

\section{Anamnese}

$\nabla$

Eine 45-jährige Patientin stellte sich mit seit Jahren bestehenden hautfarbenen, knotigen Veränderungen an der Stirn vor ( $\bullet$ Abb. 1). Als Jugendliche habe sie unter einer Akne vulgaris gelitten. Eine Therapie mit diversen lokalen Aknetherapeutika sowie Laserablation habe zu keiner Befundbesserung geführt. Allgemeinsymptome seien bisher nicht aufgetreten. Zurzeit nehme sie ein orales Kontrazeptivum und Antidepressiva ein.

\section{Hautbefund \\ $\nabla$}

An der Stirn fanden sich multiple, disseminierte etwa $3 \mathrm{~mm}$ große, derbe, weißliche bis hautfarbene Knötchen.

\section{Dermatohistopathologie $\nabla$}

Es fanden sich Exstirpate aus dem korialem Bindegewebe, bestehend aus ovalär gestaltetem Knochengewebe, z.T. trabekulär angeordnet und fokal Kalzifikationszonen enthaltend. Daneben auch komplett kalzifizierte ovaläre Strukturen (0 Abb. 2).
Primäre Osteoma cutis entwickeln sich in gesunder Haut, wohingegen sekundäre Osteoma cutis in zuvor geschädigter Haut entstehen. Die Assoziation der sekundären miliaren Osteome mit einer früheren Akne vulgaris und der erhöhten Prävalenz bei Frauen wird erklärt durch das Sexualhormon Östrogen, welches die Osteogenese fördert. Therapeutisch ist die Exstirpation der Tumore sowie ein späteres mitteltiefes, chemisches Peeling zur Narbenreduktion geplant.

\section{Laborbefunde \\ $\nabla$}

Die Autoantikörpertiter ANA und ENA waren negativ. Kreatinin, Kalzium und Phosphat zeigten Normwerte.

\section{Therapie und Verlauf \\ $\nabla$}

Es erfolgte die Exstirpation eines Teils der Tumoren in Lokalanästhesie. In weiteren Sitzungen ist die Exstirpation der noch restlichen Herde geplant. Nach Abschluss der Wundheilung ist ein mitteltiefes, chemisches Peeling zur Narbenreduktion angedacht.

\section{Diskussion \\ $\nabla$}

Osteoma cutis ist eine seltene Erkrankung, charakterisiert durch eine ausgedehnte Formation von kompaktem Knochen in der Dermis und im subkutanem Gewebe. Als klinische Differenzialdiagnostik kommen in diesem Fall in Frage: posttraumatische Läsionen, Fremdkörperreaktionen, Parasiten, Sarkoidose, Porphyria cutanea tarda, Ehlers-Danlos Syndrom, Pseudoxanthoma elasticum, Hauttumore wie Trichilemmalzysten, Pilomatrikomata, Syringome und Calcinosis Cutis. Die Diagnose miliare Osteome des Gesichtes ba- 


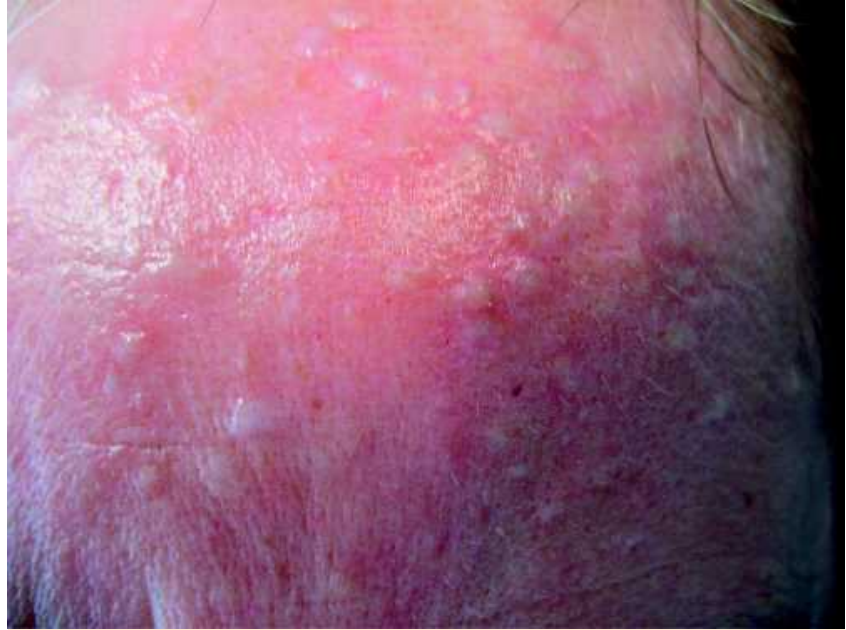

Abb. 1 Lokalbefund: multiple derbe, hautfarbene Knötchen an der Stirn.

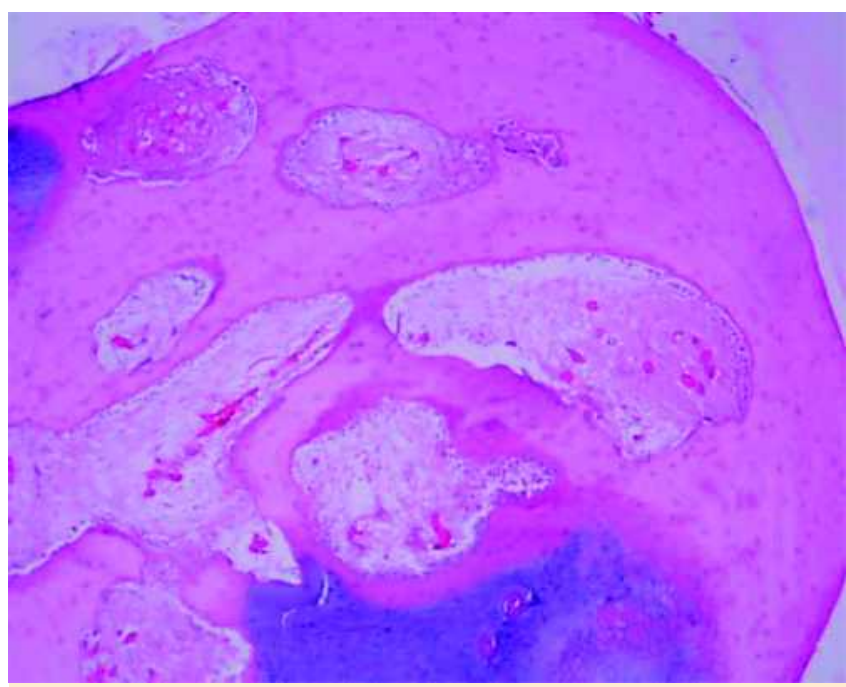

Abb. 2 Dermatohistopathologie: HE-Färbung, 100-fache Vergrößerung: Ossifikation, Kalzifikationszonen.

siert auf der typischen knöchernen Struktur in der Histologie der Knötchen.

In Abhängigkeit von der An- oder Abwesenheit von vorherigen Hautläsionen unterscheidet man zwischen primären und sekundären Formen. Primäre Osteome sind seltener, etwa 15\% der beschriebenen Fälle, und entstehen auf unveränderter Haut, z.B. bei der Albright'schen hereditären Osteodystrophie. Sekundäre Osteome sind weitaus häufiger (etwa $85 \%$ der beschriebenen Fälle) und entstehen auf vorgeschädigter Haut. Sekundäre Ossifikationen können in Narben, inflammatorischen Läsionen oder in kutanen Tumoren auftreten.

Zur Ätiologie lässt sich sagen, dass der pathogenetische Mechanismus der Entstehung von Osteomen in der Haut nicht geklärt ist. Es gibt mehrere Theorien:

Primäre Osteome können bereits bei der Geburt vorhanden sein oder sich später - wahrscheinlich aus embryonalen Zysten entwickeln. Das mesenchymale Gewebe wie Bindegewebe, Knorpel, Knochen und Fett könnten dabei eine pluripotente Fähigkeit zur Knochenbildung haben. Peterson und Mandel be- schrieben 1963 als Ursache einen gestörten embryonalen Prozess, bei dem primitive mesenchymale Zellen - also ektope Reste normaler mesenchymaler Zellen, die sich normalerweise zu Osteoblasten differenzieren, in ektoper Lokalisation (z.B. der Haut) auftreten und dort Knochen produzieren [1].

Die sekundären Osteome könnten das Ergebnis einer metaplastischen Ossifikation kalzifizierender Foci sein, z.B. an Stellen entzündlicher Narbenbildung [8]. Auch eine Metaplasie wird diskutiert, bei der mesenchymale Zellen durch unbekannte Prozesse stimuliert werden und sich so Osteoblasten ausbilden [2]. Für die sekundäre Osteoma cutis wurde eine Akne vulgaris in der Vergangenheit in einigen Fällen publiziert [1-3,7]. Dieses traf auch auf unsere Patientin zu. Die Assoziation der sekundären miliaren Osteoma Cutis mit einer früheren Akne vulgaris und der erhöhten Prävalenz bei Frauen wird erklärt durch das Sexualhormon Östrogen, welches die Knochenbildung fördert [1-3].

Die Therapie der ersten Wahl ist die operative Entfernung der Knochenfragmente oder eine Kombination mit Erbium Yag-Laser [5]. Allerdings sind auch multiple lokale und systemische Agentien versucht worden wie z.B. Tretinoin topisch [6].

\section{Abstract}

\section{Multiple Miliary Osteoma of the Face} $\nabla$

A 45-year old women presented with multiple small bony structures on the forehead exisiting for years. In her youth, she had sufferd from acne vulgaris. Histologically, miliary osteoma were confirmed. Osteoma cutis is characterized by the formation of compact bone in the skin. Primary osteoma cutis develop in healthy skin, whereas secondary osteoma cutis arise in previous cutaneous lesions. The association of secondary miliary osteoma cutis with a former acne vulgaris and the predominance of women is explained by estrogens as sexual hormones support osteogenesis. Excision of the lesions and subsequent chemical peeling are planned.

\section{Literatur}

1 Bergonse FN, Nico MM, Kavamura MI, Sotto MN. Miliary osteoma of the face: a report of 4 cases and review of the literature. Cutis 2002; 69: $383-386$

2 Stockel S, Eppinger S, Stein A, Meurer M. Multiple miliary osteomas of the face. Hautarzt 2002; 53: 37-41

3 Schuhmachers G, Worret WI. Osteoma cutis. Pathogenesis and therapeutic possibilities. Hautarzt 1992; 43: 422-425

4 Altman JF, Nehal KS, Busam KJ, Halpern AC. Treatment of primary miliary osteoma cutis with incision, curettage, and primary closure. J Am Acad Dermatol 2001; 44: 96 - 99

5 Ochsendorf FR, Kaufmann R. Erbium. Yag laser-assisted treatment of miliary osteoma cutis. Br J Dermatol 1988; 138: 371 - 372

6 Cohen AD, Chetov T, Cagnano E, Naimer S, Vardy DA. Treatment of multiple miliary osteoma cutis of the face with local application of tretinoin (all-trans-retinoic acid): a case report and review of the literature. J Dermatol Treat 2001; 12: $171-173$

7 Wagner N, Rose C, Bröcker E-B. Osteoma cutis. Akt Dermatologie 2003; 29: $87-90$

8 Moritz DL, Elewski B. Pigmented post-acne osteoma cutis in a patient treated with minocycline: report and review of the literature. J Am Acad Dermatologie 1991; 24: 851 - 853 